\title{
Reflets
}

Revue d'intervention sociale et communautaire

\section{Une campagne de sensibilisation aux réalités des communautés LGBTQ+ de la francophonie néobrunswickoise}

\section{Jean-Roch Savoie}

Volume 22, numéro 2, automne 2016

URI : https://id.erudit.org/iderudit/1038974ar

DOI : https://doi.org/10.7202/1038974ar

Aller au sommaire du numéro

Éditeur(s)

Reflets, Revue d'intervention sociale et communautaire

ISSN

1203-4576 (imprimé)

1712-8498 (numérique)

Découvrir la revue

Citer cet article

Savoie, J.-R. (2016). Une campagne de sensibilisation aux réalités des communautés LGBTQ+ de la francophonie néobrunswickoise. Reflets, 22(2), 148-154. https://doi.org/10.7202/1038974ar d'utilisation que vous pouvez consulter en ligne. 


\title{
Une campagne de sensibilisation aux réalités des communautés LGBTQ+ de la francophonie néobrunswickoise
}

\author{
Jean-Roch Savoie \\ Université de Moncton
}

Le présent article rend compte d'une pratique d'intervention communautaire conçue et mise en cuvre par deux personnes étudiant en travail social. Elle a été réalisée en milieu scolaire francophone au Nouveau-Brunswick.

\section{Un peu d'histoire... comment sommes-nous arrivés à cette campagne?}

La Fédération des jeunes francophones du Nouveau-Brunswick (FJFNB) est un organisme provincial qui porte les intérêts de la jeunesse francophone et acadienne depuis près d'un demisiècle. Cet organisme est fondé sur des principes d'inclusion, de respect et d'autodétermination, et il valorise le leadership jeunesse en ce que toutes ses activités sont réalisées par et pour les jeunes (Savoie, 2016).

Le projet dont il est ici question est issu d'une assemblée générale annuelle de la FJFNB. Chaque année, lors de leur AGA, les jeunes décident des projets majeurs qu'ils veulent réaliser au cours de la prochaine année scolaire. Or, en mai 2015, l'un de ces projets consistait à mener une campagne de sensibilisation aux réalités des communautés LGBTQ $+{ }^{1}$. Cette idée, qui provient de jeunes dont la plupart fréquentent une école secondaire, a été concrétisée par l'embauche d'une personne intervenante, en l'occurrence Jean-Roch Savoie, qui, de concert avec les autres employés de la FJFNB et son conseil d'administration jeunesse, a déterminé les objectifs de cette campagne.

Ces objectifs viennent rejoindre les principes et les valeurs des politiques 322 et 703 du ministère de l'Éducation et du Développement de la petite enfance du Nouveau- 
Brunswick. ${ }^{2}$ Ces politiques affirment l'essentialité du respect des communautés de la diversité sexuelle et de genre, et appuient les initiatives de prévention de l'intimidation et de la violence, notamment à l'endroit de l'homophobie et de la transphobie. Enfin, les principes de respect et d'appui à la prévention visent à créer un climat qui permet la célébration des diversités.

L'inclusion et la célébration des communautés francophones et acadiennes passent par la valorisation de l'ensemble des groupes qui les composent. Dans cette perspective, l'inclusion dans nos propres communautés francophones et acadiennes doit s'effectuer par une pleine reconnaissance et la valorisation des communautés de la diversité sexuelle et de genre. Par conséquent, la concrétisation de cette inclusion se réalise par la création de ressources et d'interventions par et pour les francophones en milieu minoritaire. Cette campagne lancée par la FJFNB permet de reconnaitre et de valoriser la pluralité de l'Acadie d'aujourd'hui tout en mettant l'accent sur les communautés LGBTQ+. Par surcroît, ces dernières rassemblent des groupes minoritaires qui, dans le contexte francophone et acadien du NouveauBrunswick, peuvent être doublement minorisés. Sous l'angle de l'intersectionnalité (Bilge, 2009; Simpson, 2009), la prise en compte de ces multiples identités devient particulièrement importante lorsqu'il est question de mettre en pratique la valeur de l'inclusion de l'ensemble des individus dans une visée d'équité.

À notre avis, l'importance de ce projet est profonde, puisque les communautés LGBTQ+ sont constituées d'individus, ou encore de familles, qui contribuent quotidiennement à la société, mais dont les réalités et les besoins sont souvent invisibilisés et sous-représentés, même s'ils font partie de la diversité humaine de nos communautés francophones et acadiennes.

Si une telle campagne a été mise sur pied, c'est dans le but de reconnaitre les besoins concrets des jeunes, dont les jeunes LGBTQ+, en commençant par une discussion ouverte en face à face sur l'existence et la valorisation de l'ensemble des orientations sexuelles, des sexes et des identités de genre. Ces jeunes ont aussi besoin de briser leur isolement, de sentir qu'ils appartiennent à la société, d'accéder à des personnes-ressources, voire des personnes alliées, et de se sentir en sécurité. À cet effet, des études canadiennes et américaines (Chamberland, et collab., 2011; O'Connell, et collab., 2010; Taylor, et collab., 2011) montrent que de 64 à $82 \%$ des jeunes LGBTQ+ ne se sentent pas en sécurité à l'école, ce qui vient réaffirmer l'importance de politiques provinciales telles que celles mentionnées plus haut. Le témoignage suivant ${ }^{3}$ illustre en quoi consiste le sentiment de sécurité pour un jeune en milieu scolaire rural francophone : 
"D'être safe, c'est de savoir que si j'aurai des problèmes qu'il y a quelqu'un qui va m'aider pis actually comprendre. D'être safe, c'est d'être respecté, pis de me sentir comme d'être vraiment accepté par tout le monde, pis que personne me jugerait. Il va toujours y avoir des élèves qui vont comme dire des choses, pis c'est pour ça que c'est pas vraiment $100 \%$ safe là. Ben je sais que si y'aurait des services, comme si on pourrait aller parler au travailleur social pis au psychologue, pis voir comme la direction d'école. Je ne sens pas comme si que c'est vraiment une priorité pour la direction d'école, ça devrait être plus une priorité. »

\section{Comment s'y est-on pris pour développer une campagne qui contribue à l'épanouissement de la diversité humaine?}

Cette campagne, dont les préparatifs ont débuté dès septembre 2015, était destinée à l'ensemble des 22 écoles secondaires francophones du Nouveau-Brunswick. Elle a été réalisée par le biais d'un atelier interactif intitulé « Ensemble vers le respect et l'inclusion des communautés de la diversité sexuelle et de genre ». Concrètement, cet atelier, d'une durée d'une heure, était structuré de manière à favoriser l'interaction des jeunes avec l'équipe d'animation de la FJFNB — en l'occurrence deux personnes étudiant à l'École de travail social de l'Université de Moncton - et l'interaction des jeunes entre eux. Les moyens utilisés consistaient notamment à poser diverses questions et à présenter des images pour stimuler les réflexions et les échanges. Par ailleurs, une activité basée sur la licorne du genre ${ }^{4}$ permettait aux élèves de travailler en petits groupes sur l'un des cinq concepts fondamentaux communs à l'ensemble des êtres humains, notamment l'identité de genre, l'expression de genre, le sexe biologique, l'orientation sexuelle et l'orientation romantique. Au sein de ces groupes, chaque élève devait d'abord mettre en valeur ses connaissances et ses questionnements, puis un consensus d'équipe devait s'établir sur la définition attribuée au concept assigné. Le travail effectué en petits groupes était ensuite partagé en plénière avec l'ensemble du groupe-classe. Ce partage permettait d'alimenter la discussion sur ces concepts en donnant aussi l'occasion aux personnes animatrices de corriger et de consolider les définitions des concepts. L'activité de la licorne permettait de répondre à l'un des objectifs visés, soit de sensibiliser les élèves à l'existence, à l'égalité et à la pluralité des identités de genre, des sexes, des orientations sexuelles et romantiques, etc. 


\section{L'ABC des ateliers interactifs}

Ces ateliers interactifs avaient comme objectifs de discuter des notions de respect et d'oppression engendrée par l'homophobie, la transphobie, le cissexisme et l'hétérosexisme, de prendre conscience de l'existence de personnalités publiques faisant partie des communautés LGBTQ+ et de présenter les cinq concepts fondamentaux associés à la diversité sexuelle et de genre à partir de l'image de la licorne du genre. De manière plus précise, le concept de l'identité de genre réfere au sentiment intérieur, à la perception et à l'identification de soi à l'égard de son genre (Savoie, 2016). Le sexe assigné à la naissance ne correspond pas nécessairement à l'identité de genre d'une personne. Mentionnons, à titre d'exemples, les identités de genre transgenre, cisgenre, bispirituelle, genderqueer et non binaire. En ce qui a trait au sexe assigné à la naissance, ce dernier est souvent désigné par une personne professionnelle de la santé à partir d'une évaluation visuelle des organes génitaux externes. On peut naître en tant que personne de sexe féminin ou de sexe masculin, ou encore comme personne intersexuée (Savoie, 2016). L'expression de genre, pour sa part, consiste en la façon dont chaque personne exprime, voire extériorise, son identité de genre. Par exemple, les genres peuvent être exprimés par les vêtements, le maquillage, les pronoms utilisés pour s'identifier, et cela, selon la culture à laquelle on appartient. De plus, on trouve dans la société des identités d'expression de genre comme l'identité féminine, butch, stone, masculine, agenre, etc. L'orientation romantique réfere à l'attirance et/ou l'affection sentimentale et/ou psychologique qu'une personne peut ressentir envers une autre personne en fonction du genre/sexe de celle-ci. Dans ce concept, on trouve diverses identités dont l'identité hétéromantique, panromantique, aromantique et lesboromantique (Savoie, 2016). Enfin, l'orientation sexuelle consiste en l'attirance et/ou l'affection sexuelle et/ou physique qu'une personne peut ressentir envers une autre personne en fonction du genre/sexe de celle-ci (Savoie, 2016). L'orientation sexuelle regroupe une pluralité d'identités comme l'identité gaie, bisexuelle, pansexuelle et hétérosexuelle.

\section{L'arc-en-ciel de cette campagne : valoriser le droit d'exister et d'être soi-même}

Cette campagne de la FJFNB a connu un réel succès et un beau rayonnement. Elle a donné lieu à près de 80 présentations de l'atelier interactif dans 22 écoles secondaires francophones de la province, rejoignant ainsi environ 1400 jeunes qui ont eu la chance d'y 
participer. Cette campagne a eu des retombées positives sur l'ensemble des communautés scolaires et sur les personnes qui l'ont animée. Elle a été, pour plusieurs élèves, une première occasion d'aborder l'identité de genre et/ou l'orientation romantique. Par ailleurs, la prise de conscience de l'existence de ces identités a favorisé une meilleure compréhension de la diversité humaine et de la différenciation entre orientation sexuelle et identité de genre, concepts qui sont souvent confondus. Ainsi, cet atelier a permis aux jeunes d'acquérir de nouvelles connaissances, de réfléchir sur leurs propres identités comme sur celles de leurs pairs et de prendre la parole sur la diversité sexuelle et de genre, tout en s'appropriant du vocabulaire actuel pour en parler. Le climat de sécurité établi lors des ateliers ${ }^{5}$ favorisait l'écoute et la prise de parole, où chaque élève se sentait davantage libre de s'exprimer. L'objectif d'informer et de sensibiliser les élèves sur les réalités des communautés LGBTQ+ a ainsi contribué à diminuer l'ignorance.

Cet atelier a aussi amené les jeunes à réfléchir à l'importance du respect de tous les individus, peu importe leur identité. D’ailleurs, les prises de conscience, les réflexions et les discussions ont assurément contribué à consolider le respect de la diversité humaine en général, et plus spécifiquement des communautés et des identités LGBTQ+. Par conséquent, l'ensemble de l'information et du contenu des discussions dans ces ateliers a contribué à un climat scolaire plus respectueux, inclusif et accueillant, favorisant ainsi un milieu d'apprentissage plus sécuritaire pour l'ensemble de la communauté scolaire. Ce qui est aussi porteur de changement social au sein de la communauté plus large, c'est que le sujet est porté par des jeunes qui poursuivent la discussion avec leurs proches. Certains sont même devenus des acteurs sociaux engagés dans leur milieu, par exemple en participant dans le comité LGBTQ+ et leurs alliés dans leur école. À cet effet, la FJFNB a aussi publié sur son site Internet le " Guide sur la création et la mise en œuvre d'un comité de la diversité sexuelle, de genre et leurs allié(e)s dans les écoles francophones du Nouveau-Brunswick ", un document qui a été élaboré au cours de la réalisation de la campagne (Savoie, 2016).

\section{La campagne, au-delà de l'atelier}

L'atelier consistait également à rendre visibles les communautés LGBTQ+ en offrant un kiosque de symboles et de ressources durant chaque atelier. Ce kiosque, garni aux couleurs des drapeaux des communautés asexuelles, pansexuelles et trans, entre autres, permettait aux jeunes de jeter un coup d'œil aux livres, aux films et aux symboles des communautés LGBTQ+. Le partage d'information a été essentiel pour toute personne 
qui cherchait du soutien et des renseignements ou voulait savoir comment afficher ses couleurs en tant que personne alliée.

En fin de compte, les personnes qui ont participé aux ateliers repartent non seulement avec des connaissances, mais aussi avec des outils pour faire une différence dans leur communauté. De manière plus fondamentale cependant, les jeunes qui sont en questionnement, ceux qui s'affichent comme membres des communautés LGBTQ+ ou encore les jeunes dont un membre de la famille appartient à la diversité sexuelle et de genre en sont sortis avec un sentiment de valorisation, de confiance et d'autoreconnaissance, voire de reconnaissance de son identité, de son droit d'exister. Une telle campagne provinciale a pris toute l'allure d'une intervention communautaire en milieu scolaire. Cette aventure a permis aux membres de l'équipe d'animation, en tant que personnes qui pratiqueront dans un avenir prochain le travail social, d'actualiser et de voir à l'œuvre plusieurs valeurs du travail social, notamment la poursuite de la justice sociale et le respect de la dignité, de l'unicité et de l'autodétermination des personnes, des peuples, des cultures et des communautés.

\section{Notes}

1 L'acronyme LGBTQ+ a comme signification les identités lesbienne, gaie, bisexuelle, transgenre, queer, en questionnement, et plus encore.

2 Les politiques 322 et 703 du ministère de l'Éducation et du Développement de la petite enfance se trouvent en ligne aux adresses suivantes :

http://www2.gnb.ca/content/dam/gnb/Departments/ed/pdf/K12/policies-politiques/f/322F.pdf http://www2.gnb.ca/content/dam/gnb/Departments/ed/pdf/K12/policies-politiques/f/703F.pdf

3 Ce témoignage a été obtenu pour une recherche que la personne auteure du présent article est en train de compléter dans le cadre de ses études de maîtrise en travail social. Son projet s'intitule, de manière provisoire : "Comprendre les réalités vécues par les jeunes témoins et/ou victimes de transphobie, d'homophobie, d'hétérosexisme et/ou de cissexisme afin de consolider un milieu propice à l'apprentissage et inclusif à l'ensemble de la communauté scolaire ».

4 La licorne du genre a été produite par Landyn Pan de l'organisme Trans Student Educational Resources (TSER), dont le site Web est à l'adresse suivante : www.transstudent.org/gender

5 Au début de chaque atelier, l'équipe d'animation présentait un code de vie afin d'assurer un climat sécuritaire et respectueux pour l'ensemble des élèves. 


\section{Bibliographie}

BILGE, Sirma (2009). "Théorisation féministe de l'intersectionalité », Diogène, No 225, p. 70-88.

CHAMBERLAND, Line, et collab. (2011). La transphobie en milieu scolaire au Québec, [rapport de recherche], Montréal, Université du Québec à Montréal, 39 p.

CHAMBERLAND, Line, et collab. (2011). L’homophobie à l'école secondaire au Québec. Portrait de la situation, impacts et pistes de solution, [rapport de recherche], Montréal, Université du Québec à Montréal, $29 \mathrm{p}$.

O’CONNELL, Lynn, et collab. (2010). « Perceptions of Rural School Staff Regarding Sexual Minority Students ", Journal of LGBT Youth, Routledge: Taylor and Francis Group, Vol.7, № 4, p. 293-309.

SAVOIE, Jean-Roch (2016). Guide sur la création et la mise en ouvre d'un comité de la diversité sexuelle, de genre et leurs allié(e)s dans les écoles francophones du Nouveau-Brunswick, Fédération des jeunes francophones du Nouveau-Brunswick, Moncton, NouveauBrunswick, réf. du 3 septembre 2016, http://www.fjfnb.nb.ca/sites/default/files/documents/outil/739/guidediversitesexuellefjfnbweb. pdf

SIMPSON, Joanna (2009). Everyone Belongs. A Toolkit for Applying Intersectionality, Ottawa, Institut canadien de recherches sur les femmes.

TAYLOR, Catherine, et collab. (2011). "Every class in every school: The first national climate survey on homophobia, biphobia, and transphobia in Canadian schools", Rapport final, Toronto, Egale Canada Human Rights Trust, 152 p. 